\title{
1 New Asgard archaea capable of anaerobic hydrocarbon cycling
}

2 Kiley W. Seitz ${ }^{1}$, Nina Dombrowski ${ }^{1,3}$, Laura Eme ${ }^{2}$, Anja Spang ${ }^{2,3}$, Jonathan Lombard ${ }^{2}$, Jessica R. Sieber ${ }^{4}$,

3 Andreas P. Teske ${ }^{5}$, Thijs J.G. Ettema ${ }^{2}$, and Brett J. Baker ${ }^{1^{*}}$

4 1. Department of Marine Science, University of Texas Austin, Port Aransas, TX 78373 2. Uppsala University, 5 Uppsala Sweden 3. NIOZ, Royal Netherlands Institute for Sea Research, and Utrecht University, The 6 Netherlands 4. University Minnesota Duluth, MN 5. Department of Marine Sciences, University of North 7 Carolina, Chapel Hill, NC *Corresponding author

Large reservoirs of natural gas in the oceanic subsurface sustain a complex biosphere of

11 anaerobic microbes, including recently characterized archaeal lineages that extend the

12 potential to mediate hydrocarbon oxidation (methane and butane) beyond the

13 Methanomicrobia. Here we describe a new archaeal phylum, Helarchaeota, belonging to the

14 Asgard superphylum with the potential for hydrocarbon oxidation. We reconstructed

15 Helarchaeota genomes from hydrothermal deep-sea sediment metagenomes in hydrocarbon-

16 rich Guaymas Basin, and show that these encode novel methyl-CoM reductase-like enzymes

17 that are similar to those found in butane-oxidizing archaea. Based on these results as well as

18 the presence of several alkyl-CoA oxidation and Wood-Ljungdahl pathway genes in the

19 Helarchaeota genomes, we suggest that members of the Helarchaeota have the potential to

20 activate and subsequently anaerobically oxidize short-chain hydrocarbons. These findings link

21 a new phylum of Asgard archaea to the microbial utilization of hydrothermally generated

22 hydrocarbons, and extend this genomic blueprint further through the archaeal domain. 
25 Short-chain alkanes, such as methane and butane, are abundant in marine sediments and play

26 an important role in carbon cycling with methane concentrations of $\sim 1$ Gt being processed

27 globally through anoxic microbial communities ${ }^{1-3}$. Until recently, archaeal methane cycling was

28 thought to be limited to Euryarchaeota ${ }^{4}$. However, additional archaeal phyla, including

29 Bathyarchaeota ${ }^{5}$ and Verstraetarchaeota ${ }^{6}$, have been shown to contain proteins with homology

30 to the activating enzyme methyl-coenzyme $\mathrm{M}$ reductase (Mcr) and corresponding pathways for

31 methane utilization. Furthermore, new lineages within the Euryarchaeota belonging to

32 Candidatus Syntrophoarchaeum spp., have been shown to use methyl-CoM reductase-like

33 enzymes for anaerobic butane oxidation ${ }^{7}$. Similar to methane oxidation in many ANME-1 archaea,

34 butane oxidation in Syntrophoarchaeum is proposed to be enabled through a syntrophic

35 interaction with sulfur reducing bacteria ${ }^{7}$. Metagenomic reconstructions of genomes recovered

36 from deep-sea sediments from near 2000 m depth in Guaymas Basin (GB) in the Gulf of California

37 have revealed the presence of additional uncharacterized alkyl methyl-CoM reductase-like

38 enzymes in metagenome-assembled genomes within the Methanosarcinales (Gom-Arc1) ${ }^{8}$. GB is

39 characterized by hydrothermal alterations that transform large amounts of organic carbon into

40 methane, polycyclic aromatic hydrocarbons (PAHs), low-molecular weight alkanes and organic

41 acids allowing for diverse microbial communities to thrive (Supplementary Table 1$)^{8-11}$.

Recently, genomes of novel clade of uncultured archaea, referred to as the Asgard

43 superphylum that includes the closest archaeal relatives of eukaryotes, have been recovered

44 from anoxic environments around the world ${ }^{12-14}$. Diversity surveys in anoxic marine sediments

45 show that Asgard archaea appear to be globally distributed ${ }^{9,11,12,13}$. Based on phylogenomic

46 analyses, Asgard archaea have been divided into four distinct phyla: Lokiarchaeota, 
47 Thorarchaeota, Odinarchaeota and Heimdallarchaeota, with the latter possibly representing the

48 closest relatives of eukaryotes ${ }^{12}$. Supporting their close relationship to eukaryotes, Asgard

49 archaea possess a wide repertoire of proteins previously thought to be unique to eukaryotes

50 known as eukaryotic signature proteins (ESPs) ${ }^{17}$. These ESPs include homologs of eukaryotic

51 proteins, which in eukaryotes are involved in ubiquitin-mediated protein recycling, vesicle

52 formation and trafficking, endosomal sorting complexes required for transport (ESCRT)-mediated

53 multivesicular body formation as well as cytokinetic abscission and cytoskeleton formation ${ }^{18}$.

54 Asgard archaea have been suggested to possess heterotrophic lifestyles and are proposed to play

55 a role in carbon degradation in sediments; however, several members of the Asgard archaea also

56 have genes that code for a complete Wood-Ljungdahl pathway and are therefore interesting with

57 regard to carbon cycling in sediments ${ }^{14,19}$.

58 Here we present the first evidence of metagenome assembled genomes (MAGs),

59 recovered from Guaymas Basin deep-sea hydrothermal sediments, which represent a new

60 Asgard phylum with the metabolic potential to perform anaerobic hydrocarbon degradation

61 using a methyl-CoM reductase-like homolog.

\section{Results}

64 Identification of Helarchaeota genomes from Guaymas Basin sediments. We recently obtained

65 over 280 gigabases of sequencing data from 11 samples taken from various sites and depths at

66 Guaymas Basin hydrothermal vent sediments ${ }^{20}$. De novo assembly and binning of metagenomic

67 contigs resulted in the reconstruction of over 550 genomes ( $>50 \%$ complete) ${ }^{20}$. Within these

68 genomes we detected a surprising diversity of archaea, including $>20$ phyla, which appear to 
69 represent up to $50 \%$ of the total microbial community in some of these samples ${ }^{20}$. Preliminary

70 phylogeny of the dataset using 37 concatenated ribosomal proteins revealed two draft genomic

71 bins representing a new lineage in the Asgard archaea. These draft genomes, referred to as

72 Hel_GB_A and Hel_GB_B, were re-assembled and re-binned resulting in final bins that were 82

73 and $87 \%$ complete and had a bin size of 3.54 and $3.84 \mathrm{Mbp}$, respectively (Table 1). An in-depth

74 phylogenetic analysis consisting of 56 concatenated ribosomal proteins was used to confirm the

75 placement of these final bins form a distant sister-group with the Lokiarchaeota (Figure 1a).

76 Hel_GB_A percent abundance ranged from $3.41 \times 10^{-3} \%$ to $8.59 \times 10^{-5} \%$ and relative abundance from 8.43

77 to 0.212 . Hel_GB_B percent abundance ranged from $1.20 \times 10^{-3} \%$ to $7.99 \times 10^{-5} \%$ and relative abundance

78 from 3.41 to 0.22 . For both Hel_GB_A and Hel_GB_B the highest abundance was seen at the site the

79 genomes bins were recovered from. These numbers are comparable to other Asgard archaea isolated

80 form these $\operatorname{sites}^{20}$. Hel_GB_A and Hel_GB_B had a mean GC content of $35.4 \%$ and $28 \%$, respectively,

81 and were recovered from two distinct environmental samples, which share similar methane-

82 supersaturated and strongly reducing geochemical conditions (concentrations of methane

83 ranging from 2.3-3 mM, dissolved inorganic carbon ranging from 10.2-16.6 mM, sulfate near 21

$84 \mathrm{mM}$ and sulfide near $2 \mathrm{mM}$; Supplementary Table 1$)$ but differed in temperature $\left(28^{\circ} \mathrm{C}\right.$ and $10^{\circ} \mathrm{C}$,

85 respectively, Supplementary Table 1$)^{19}$.

86 Phylogenetic analyses of a 16S rRNA gene sequence (1058 bp in length) belonging to

87 Hel_GB_A confirmed that they are related to Lokiarchaeota and Thorarchaeota, but are

88 phylogenetically distinct from either of these lineages (Figure 1b). A comparison to published

89 Asgard archaeal 16S rRNA gene sequences indicate a phylum level division between the

90 Hel_GB_A sequence and other Asgard $\operatorname{archaea}^{22}$ (Supplementary Table 2). A search for ESPs in 
91 both bins revealed that they contained a similar suite compared to those previously identified in

92 Lokiarchaeota, which is consistent with their distant phylogenomic relationship (Figure 2). These

93 lineages are relatively distantly related as evidenced by their difference in GC content and

94 relatively low pairwise sequence identity of proteins. An analysis of the average amino acid

95 identity (AAI) showed that Hel_GB_A and Hel_GB_B shared 1477 genes with and AAl of 51.96\%.

96 When compared to Lokiarcheota_CR4, Hel_GB_A share 634 out of orthologous genes 3595 and

97 Hel_GB_B had 624 orthologous genes out of 3157. Helarchaeota bins showed the highest AAl

98 similarity to Odinarchaeota LCB_4 (45.9\%); however, it contained fewer orthologous genes (574

99 out of 3595 and 555 out of 3157 for Hel_GB_A and Hel_GB_B, respectively). Additionally, the

100 Hel_GB bins differed from Lokiarchaeota in their total gene number, for example Hel_GB_A

101 possessed 3595 genes and CR_4 possessed 4218; this difference is consistent with the larger

102 estimated genome size for Lokiarchaeum CR_4 compared to Hel_GB_A ( 5.2 Mbp to 4.6 Mbp)

103 (Supplementary Table 3, Supplementary Methods). These results add support to the phylum level

104 distinction observed for Hel_GB_A and Hel_GB_B in both the ribosomal protein and 16s rRNA

105 phylogenetic trees. We propose the name Helarchaeota after Hel, the Norse goddess of the

106 underworld and Loki's daughter for this lineage. 
bioRxiv preprint doi: https://doi.org/10.1101/527697; this version posted January 24, 2019. The copyright holder for this preprint (which was not certified by peer review) is the author/funder. All rights reserved. No reuse allowed without permission.

A.

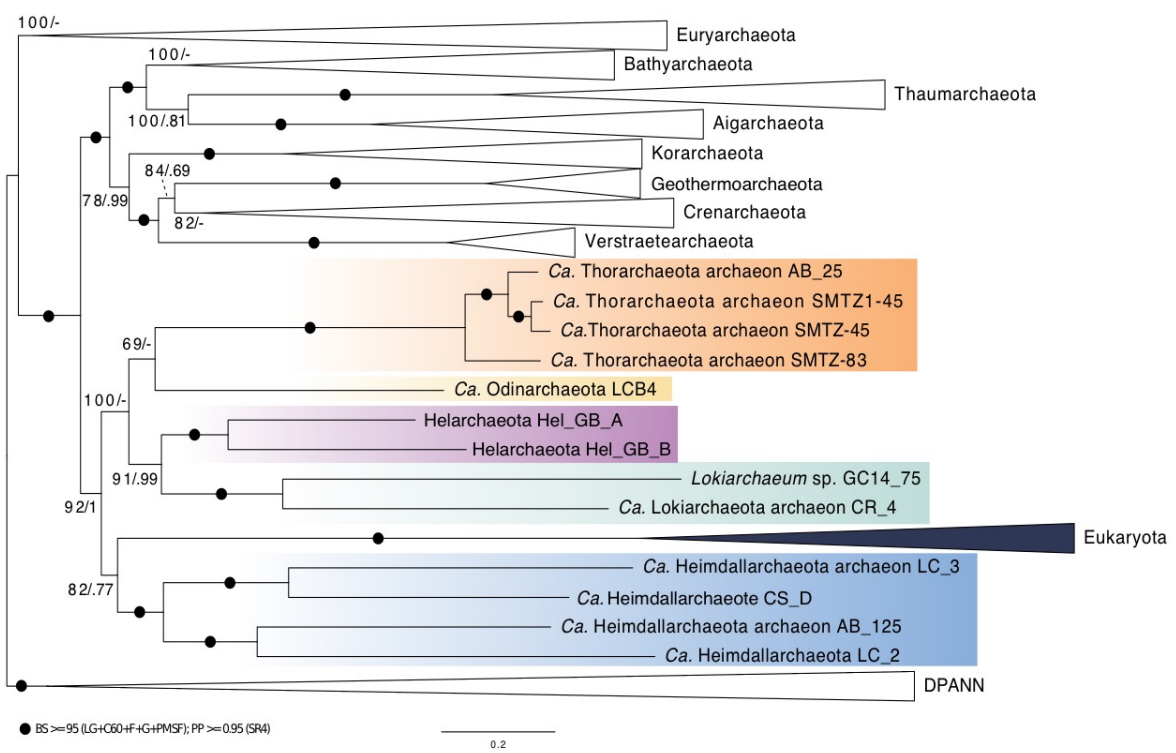

B.

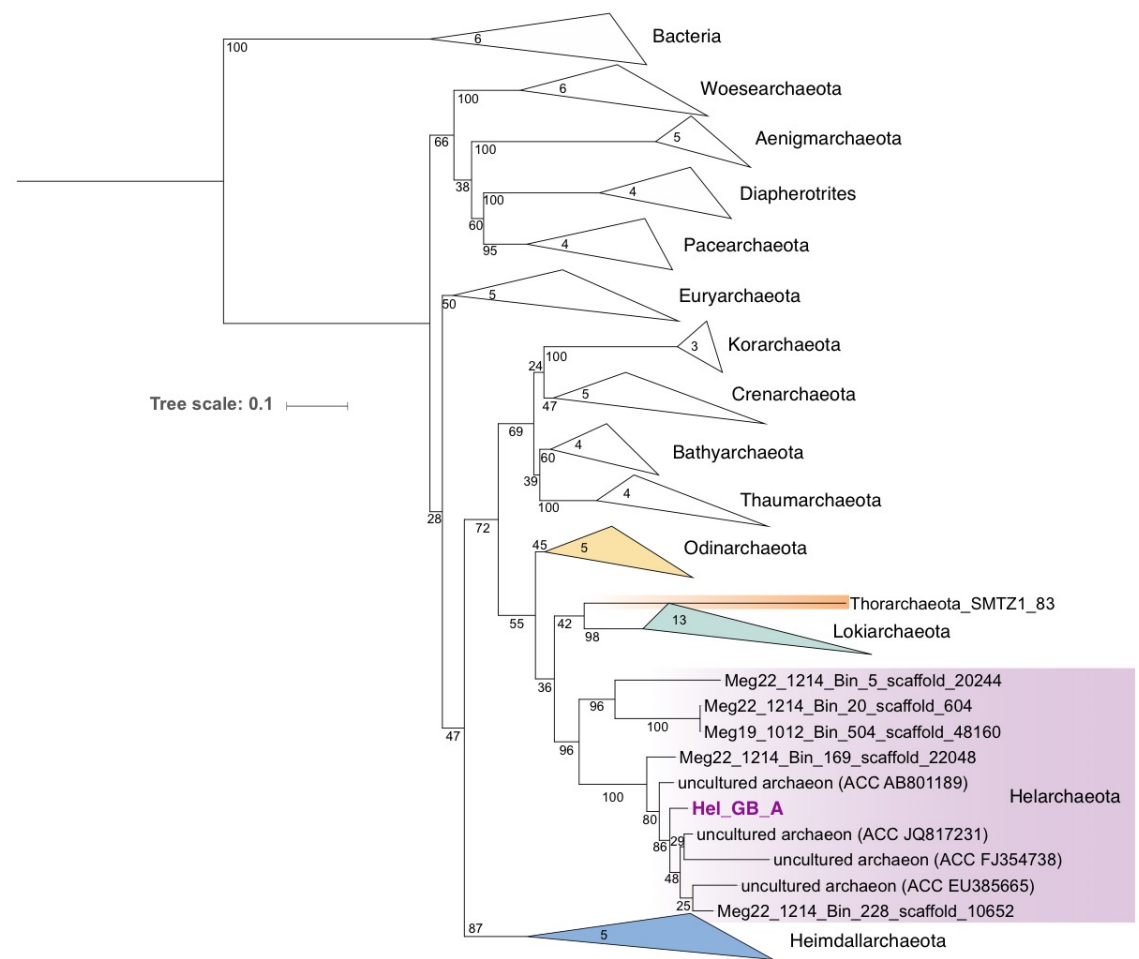

108 Figure 1. Phylogenomic position of Helarchaeota within the Asgard archaea superphylum (A)

109 Phylogenomic analysis of 56 concatenated ribosomal proteins identified in Helarchaeota bins. Black circles

110 indicate Bootstrap values greater than 95 (LG+C60+F+G+PMSF); Posterior Probability >= 0.95 (SR4). (B)

111 Maximum-likelihood phylogenetic tree of 16S rRNA gene sequences thought to belong to Helarchaeota. 
112 The phylogeny was generated using RAxML (GTRGAMMA model and number of bootstraps determined

113 using the extended majority-rule consensus tree criterion). The purple box shows possible Helarchaeota

114 sequences from GB data, as well as closely related published sequences and sequences form newly

115 identified Helarchaeota bins (identified as Megxx_xxxx_Bin_xxx_scaffold_xxxxx). Number of sequences is

116 depicted in the closed branches.

118 Metabolic analysis of Helarchaeota. To reconstruct the metabolic potential of these archaea,

119 the Helarchaeota proteomes were compared to several functional protein databases ${ }^{20}$ (Figure

120 3a). Like many archaea in marine sediments ${ }^{23}$, Helarchaeota may be able to utilize organic carbon

121 as they possess a variety of extracellular peptidases and carbohydrate degradation enzymes that

122 include the $\beta$-glucosidase, $\alpha$-L-arabinofuranosidase and putative rhamnosidase, among others

123 (Supplementary Table 4 and 5). Degraded organic substrates can then be metabolized via

124 glycolysis and an incomplete TCA cycle from citrate to malate and a partial gamma-aminobutyric

125 acid shunt (Figure 3a, Supplementary Table 4). Both Helarchaeota bins are missing fructose-1,6-

126 bisphosphatase and have few genes coding for the pentose phosphate pathway. Genes encoding

127 for the bifunctional enzyme 3-hexulose-6-phosphate synthase/6-phospho-3-hexuloisomerase

128 (hps-phi) were identified in Hel_GB_B suggesting they may be using the ribulose monophosphate

129 (RuMP) pathway for formaldehyde anabolism. Genes coding for acetate-CoA ligase (both APM

130 and ADP-forming) and an alcohol dehydrogenase (adhE) were identified in both genomes

131 suggesting that the organisms may be capable of both fermentation and production of acetyl-

132 CoA using acetate and alcohols (Supplementary Table 4). Like in Thorarchaeota and

133 Lokiarchaeota, these genomes possess the large subunit of type IV Ribulose bisphosphate

134 carboxylase ${ }^{19,24}$. Additionally, the Helarchaeota genomes encode for the catalytic subunit of the 
135 methanogenic type III ribulose bisphosphate carboxylase used for C-fixation ${ }^{24}$. Helarchaeota are

136 metabolically distinct from Lokiarchaeota as both Hel_GB draft genomes appear to lack a

137 complete TCA cycle as genes coding for citrate synthase and malate/lactate dehydrogenase are

138 absent. Both genomes also likely produce acetyl-CoA using glyceraldehyde 3-phosphate

139 dehydrogenase which is absent in Lokiarchaeota $^{19}$ (Supplementary Table 4). Helarchaeota

140 genomes lack genes that code for enzymes involved in dissimilatory nitrogen and sulfur

141 metabolism. Assimilatory genes including sat, cysN and cysC were found in Hel_GB_B however

142 these genes were not identified in Hel_GB_A. This absence may be indicative of species-specific

143 characteristics of their genomes or could be a results of genome incompleteness. Additional

144 genomes of members of the Helarchaeota will help to fully understand the diversity of these

145 pathways across the whole phylum.

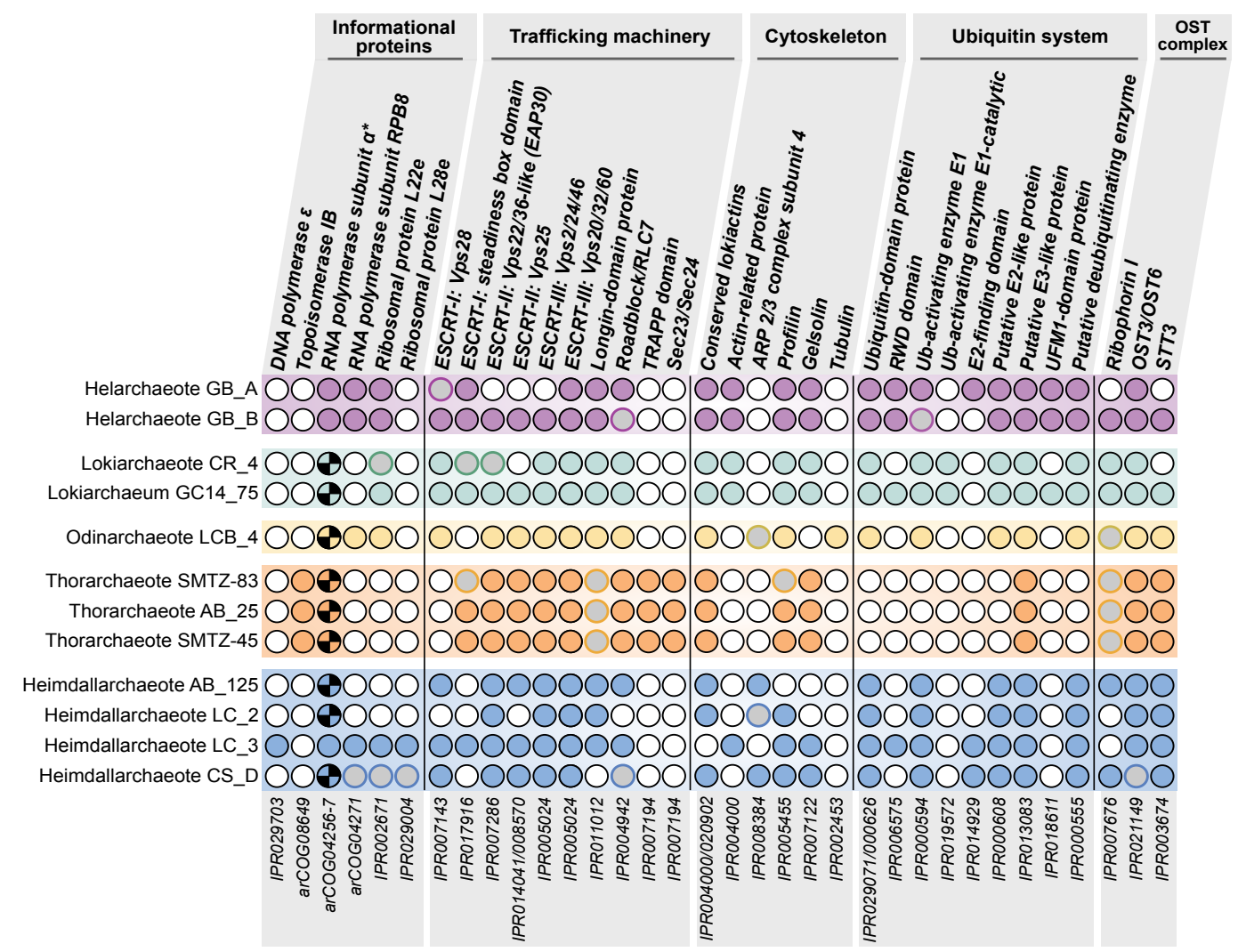


148 Numbers under each column correspond to the InterPro accession number (IPR) and Archaeal Clusters of 149 Orthologous Genes (arcCOG) IDs that were searched for. Full circles refer to cases in which a homologue was found in the respective genomes. Empty circles with black outlines represent the absence of the ESP.

151 The checkered pattern in the RNA polymerase subunit alpha represents the fact that the proteins were 152 split, while the fused proteins are represented by the full circles. Grey circles with borders in any other 153 color represent cases where the standard profiles were not found but potential homologs where detected.

154 In the Roadblock proteins, potential homologs were detected but the phylogeny could not support the 155 close relationship of any of these copies to the Asgard archaea group closest to eukaryotes. In the Ubactivating enzyme E1 represents homologs found clustered appropriately with its potential orthologs in

157 the phylogeny but the synteny of this gene with other ubiquitin-related proteins in the genome is 158 uncertain.

Interestingly, both Helarchaeota genomes have mcrABG-containing gene clusters encoding putative methyl-CoM reductase-like enzymes (Figure 3b, Supplementary Figure 2) (,5,7. $^{4}$

162 Phylogenetic analyses of both the A subunit of methyl-CoM reductase-like enzymes

163 (Supplementary Figure 2) as well as the concatenated A and B subunits (Figure 3b) revealed that

164 the Helarchaeota sequences are distinct from those involved in methanogenesis and methane 165 oxidation but cluster with homologs from butane oxidizing Syntrophoarchaea ${ }^{7}$ and 166 Bathyarchaeota with high statistical support (rapid bootstrap support/single branch test 167 bootstrap support/posterior probability of 99.8/100/1; Figure 3b) excluding the distant homolog 168 of Ca. Syntrophoarchaeum caldarius (OFV68676). Analysis of the Helarchaeota mcrA alignment 169 confirmed that amino acids present at their active sites are similar to those identified on 
170 Bathyarchaeota and Syntrophoarchaeum methyl-CoM reductase-like enzymes (Supplementary

171 Figure 3). In Syntrophoarchaeum, the methyl-CoM reductase-like enzymes have been suggested

172 to activate butane to butyl-CoM${ }^{7}$. It is proposed that this process is then followed by the

173 conversion of butyl-CoM to butyryl-CoA; however, the mechanism of this reaction is still

174 unknown. Butyryl-CoA can then be oxidized to acetyl-CoA that can be further feed into the Wood-

175 Ljungdahl pathway to produce $\mathrm{CO}_{2}{ }^{7}$. While some n-butane is detected in Guaymas Basin

176 sediments (usually below 10 micromolar), methane is the most abundant hydrocarbon

177 (Supplementary Table 1) followed by ethane and propane (often reaching the 100 micromolar

178 range); thus, a spectrum of short-chain alkanes could potentially be metabolized by

179 Helarchaeota26.

A.

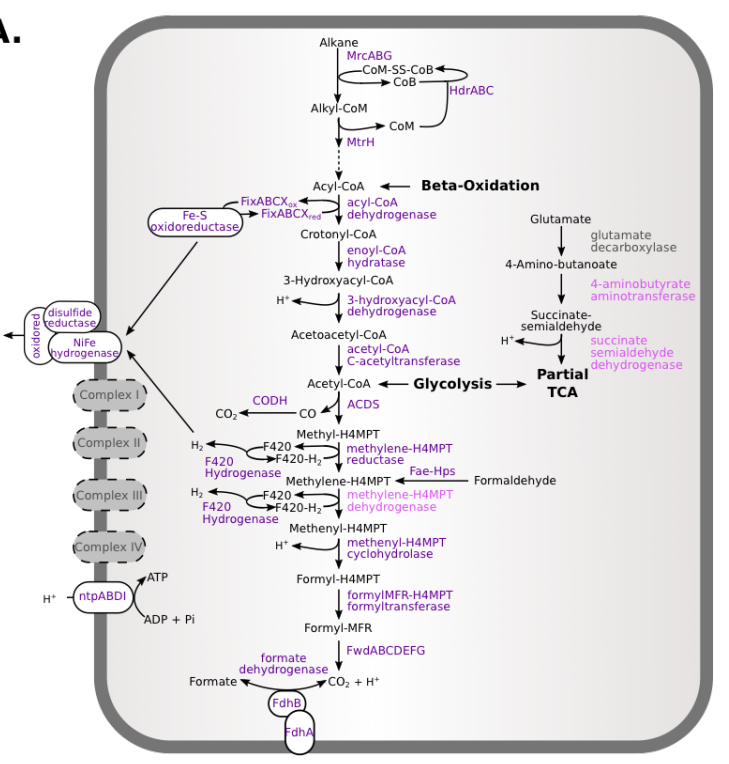

B.

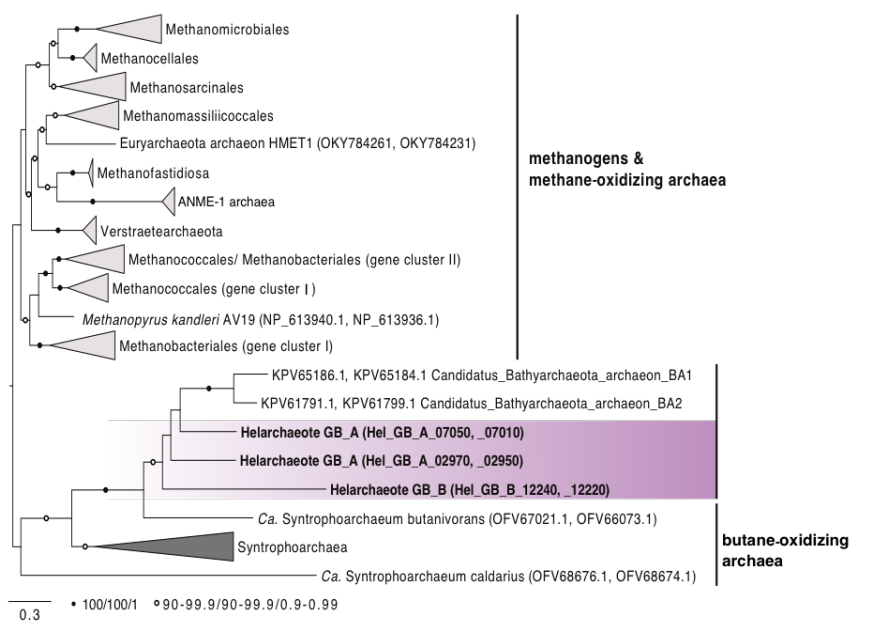

181 Figure 3. Metabolic inference of Helarchaeota and phylogenetic analyses of concatenated McrAB

182 proteins. (A) Enzymes shown in dark purple are present in both genomes, those shown in light purple are

183 present in a single genome and ones in grey are absent. (B) The tree was generated using IQ-tree with

1841000 ultrafast bootstraps, single branch test bootstraps and posterior predictive values from the Bayesian

185 phylogeny. White circles indicate bootstrap values of 90-99.9/90-99.9/0.9-0.99 and black filled circles 
indicate values of $100 / 100 / 1$. The tree was rooted arbitrarily between the cluster comprising canonical McrAB homologs and divergent McrAB homologs, respectively. Scale bars indicate the average number of substitutions per site.

Proposed hydrocarbon degradation pathway for Helarchaeota. Next, we searched for genes

191 encoding enzymes potentially involved in hydrocarbon utilization pathways including propane

192 and butane oxidation. Along with the methyl-CoM reductase-like enzyme that could convert

193 alkane to alkyl-CoM, Helarchaeota possess heterodisulfide reductase subunits $A B C(h d r A B C)$

194 which is needed to recycle the CoM and CoB heterodisulfides after this reaction occurs (Figure 3

195 and 4$)^{7,8}$. The conversion of alkyl-CoM to acyl-CoA is currently not understood in archaea capable

196 of butane oxidation. Novel alkyl-binding versions of methyltransferases would be required to

197 convert alkyl-CoM to butyl-CoA or other acyl-CoAs, as discussed for Ca. S. butanivorans ${ }^{7}$. Genes

198 coding for methyltransferases were identified in both Helarchaeota genomes, including a likely

199 tetrahydromethanopterin S-methyltransferase subunit $\mathrm{H}$ (MtrH) homolog (Figure 4;

200 Supplementary Table 4). Short-chain acyl-CoA could be oxidized to acetyl-CoA using the beta-

201 oxidation pathway via a short-chain acyl-CoA dehydrogenase, enoyl-CoA hydratase, 3-

202 hydroxyacyl-CoA dehydrogenase and acetyl-CoA acetyltransferase, candidate enzymes for all of

203 which are present in the Helarchaeota genomes and are also found in genomes of other Asgard

204 archaea (Figure 4) ${ }^{19}$. Along with these enzymes, genes coding for the associated electron transfer

205 systems, including an Fe-S oxidoreductase and all subunits of the electron transfer flavoprotein

206 (ETF) complex were identified in Helarchaeota (Figure 4). Acetyl-CoA produced by beta-oxidation

207 might be further oxidized to $\mathrm{CO}_{2}$ via the Wood-Ljungdahl pathway, using among others the 


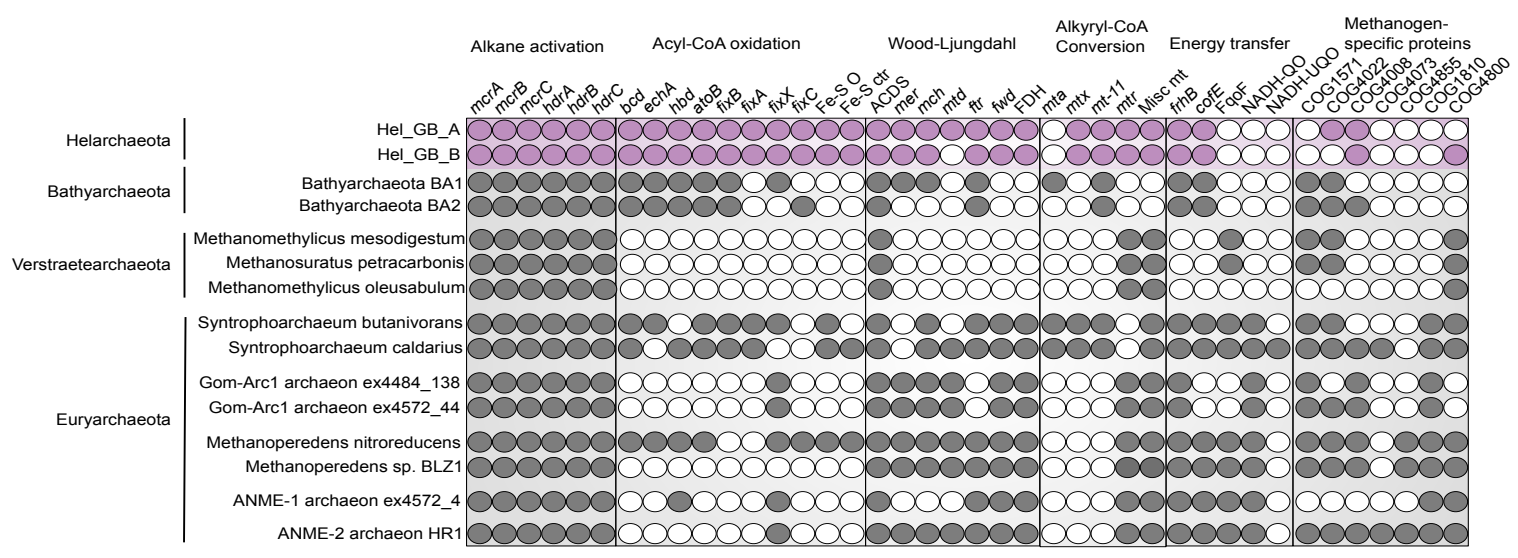

211 Figure 4. Comparison of Helarchaeota alkane metabolism to other alkane oxidizing and methanogenic

212 archaea. Alkane metabolism of Helarchaeota compared to Bathyarchaeota and Ca. Syntrophoarchaeum

213 sp., Verstraetearchaeota, GoM-Arc1 sp., ANME-1 sp. and ANME-2 sp. A list of genes and corresponding

214 contig identifiers can be found in Supplementary Table 4.

216 Three possible energy-transferring mechanisms for Helarchaeota. To make anaerobic alkane

217 oxidation energetically favorable, it must be coupled to the reduction of an internal electron

218 acceptor or transferred to a syntrophic partner that can perform this reaction ${ }^{7,26,27}$. We could not

219 identify an internal electron sink or any canonical terminal reductases used by ANME archaea

220 (such as iron, sulfur or nitrogen), leading to the conclusion that a syntrophic partner organism

221 would be necessary to enable growth on short-chain hydrocarbons. However, we could not

222 identify any obvious syntrophic partner organisms based on co-occurrence analyses of

223 abundance profiles of metagenomic datasets generated in this study ${ }^{20}$. 
An evaluation of traditional energy transferring mechanisms showed that our

225 Helarchaeota bins lack genes coding for NADH:ubiquinone oxidoreductase, $\mathrm{F}_{420}$-dependent

226 oxidoreductase, $\mathrm{F}_{420} \mathrm{H}_{2}$ :quinone oxidoreductase and $\mathrm{NADH}$ :quinone oxidoreductase that were

227 identified in Ca. S. butanivorans (Figure 4) $)^{7}$. These electron-carrying proteins are important for

228 energy transfer across the cell membrane and are common among syntrophic organisms ${ }^{2,28,29}$.

229 Helarchaeota also lack genes coding for pili or cytochromes that are generally associated with

230 electron transfer to a bacterial partner, as demonstrated for different ANME $\operatorname{archaea}^{26,30}$.

231 Therefore, Helarchaeota may use a thus far unknown approach for energy conservation. Below

232 we analyzed potential energy-transferring mechanisms that might be involved in syntrophic

233 interactions between Helarchaeota and potential partner organisms.

A possible candidate for energy transfer to a partner may be formate dehydrogenase

235 because substrate exchange in form of formate has previously been described to occur between

236 methanogens and sulfur-reducing bacteria ${ }^{27}$. Helarchaeota genomes code for the alpha and beta

237 subunits of a membrane-bound formate dehydrogenase (EC. 1.2.1.2) that could facilitate this

238 transfer (Figure 2, Supplementary Table 4). However, to our knowledge formate transfer has not

239 been shown to mediate methane oxidation. Alternatively, Helarchaeota may possess a novel

240 redox-active complex. In both Helarchaeota bins, a gene cluster was found encoding three

241 proteins that were identified as members of the HydB/Nqo4-like superfamily, Oxidored_q6

242 superfamily and a Fe-S disulfide reductase with a FlpD domain (mvhD) (Figure 5a). An analysis of

243 these three proteins showed that each possessed transmembrane motifs (Figure 5b, and

244 Supplementary Methods). While the membrane association of the disulfide reductase/FlpD 
245 needs to be confirmed, interactions with the other two membrane-associated subunits may

246 allow for the bifurcated electrons to be transferred across the membrane.

$247 \quad$ Finally, hydrogen production and release was also considered as possible electron sink for

248 Helarchaeota. We identified several hydrogenases and putative Fe-S disulfide reductase-

249 encoding genes in the Helarchaeota genomes. Subsequent phylogenetic analyses revealed that

250 the majority of these hydrogenases represent small and large subunits of group IIIC hydrogenases

251 (methanogenic $\mathrm{F}_{420}$-non-reducing hydrogenase $(m v h)$ ) that are usually involved in bifurcating

252 electrons from hydrogen (Supplementary Figure 4, Supplementary Table 4). In contrast, while

253 homologs belonging to the above mentioned Oxidored_q6 superfamily protein family are often

254 found to be associated with group IV hydrogenases, canonical membrane-bound group IV-

255 hydrogenases could not be identified in the genomes of the Helarchaeota. Altogether, this

256 indicates that hydrogen could play a central role in energy metabolism of Helarcharota, but the

257 absence of a classical membrane-bound hydrogenase makes it unlikely that hydrogen is the 258 major syntrophic electron carrier. 


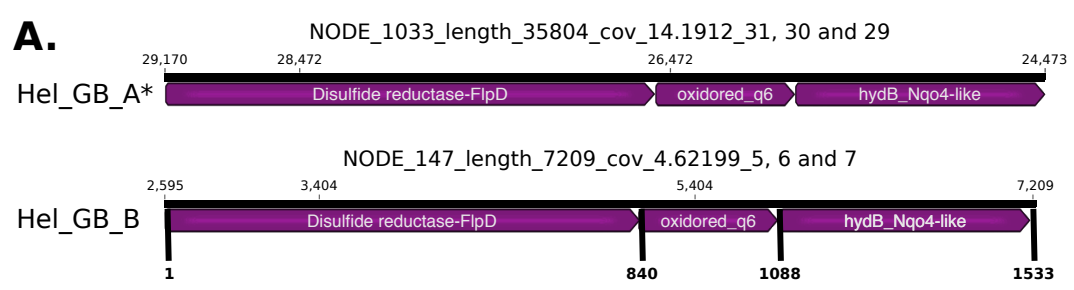

B. Hel_GB_B

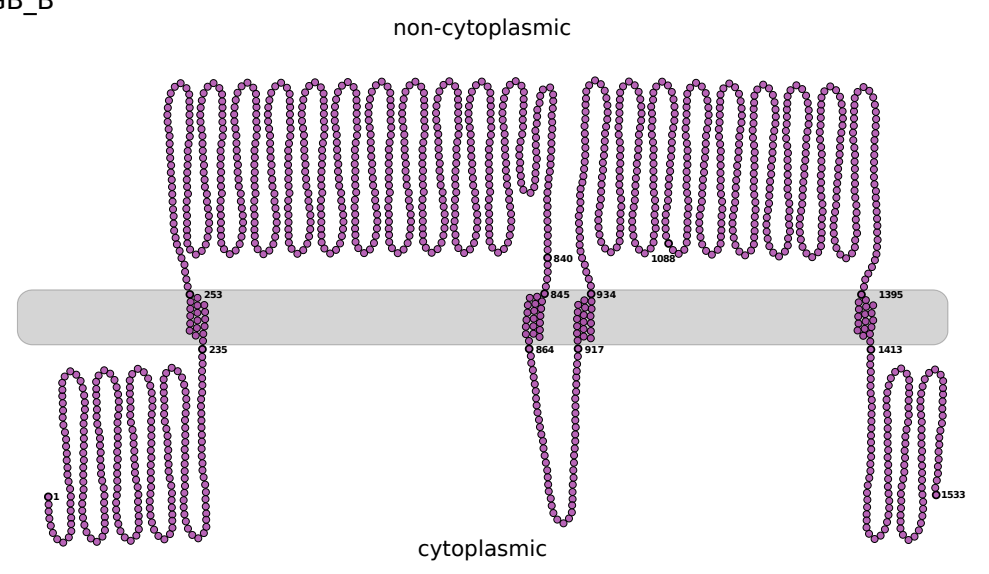

Figure 5. Depiction of a gene cluster found in both Helarchaeota genomes that consists of genes that encode for a possible energy-transferring complex. (A) In Hel_GB_A the complex was found on the reverse strand but has been oriented in the forward direction for clarity (asterisk). Arrows indicate the length of the reading frame. Gene names were predicted by various databases (Supplementary Methods).

264 Small numbers located above the arrows refer to the nucleotide position for the full contig. Bold numbers on Hel_GB_B refer to the amino acid number of the whole complex. (B) Figure depicts the membrane motifs identified on NODE_147_length_7209_cov_4.62199_5, 6 and 7 using various programs

267 (Supplementary methods). Each circle represents a single amino acid. Bold circles represent amino acids at the start of the protein, the start and end of the transmembrane sites, and the end of the complex. amino acids and does not reflect the secondary structure of the complex. 


\section{Discussion}

273 Historically methanogenesis and anaerobic methane oxidation were regarded as the only

274 examples of anaerobic archaeal short-chain alkane metabolism. The enzymes acting in these

275 pathways were considered to be biochemically and phylogenetically unique and limited to

276 lineages within the Euryarchaeota ${ }^{4}$. This study represents the discovery of a novel phylum and

277 the first indications for anaerobic short-chain alkane oxidation using a MCR-like homolog in the

278 Asgard archaea. Since the presence of these mor genes is restricted to Helarchaeota among the

279 known Asgard archaea ${ }^{19}$, these genes were likely transferred to Helarchaeota and do not

280 constitute an ancestral trait within the Asgard superphylum. Based on current phylogenetic

281 analysis, the Helarchaeota $\mathrm{mcr}$ gene cluster may have been horizontally acquired from either

282 Bathyarchaeota or Ca. Syntrophoarchaeum (Fig. 1b, Supplementary Figure 3). Due to this close

283 relationship, we based our analysis of Helarchaeota's ability to perform anaerobic short-chain

284 hydrocarbon oxidation on the pathway proposed for Ca. Syntrophoarchaeum. Helarchaeota

285 probably utilize a similar short-chain alkane as a substrate in lieu of methane, but given the low

286 butane concentrations at our site it may not be an exclusive substrate.

288 for this metabolism including a complete Wood-Ljungdahl pathway, acyl oxidation pathway and

289 internal electron transferring systems. These electron-transferring systems are essential

290 housekeeping components that act as electron carriers for oxidation reactions. Interestingly, in

291 the Wood-Ljungdahl pathway identified in Ca. S. butanivorans, the bacterial enzyme is 5,10-

292 methylene-tetrahydrofolate reductase (met) is thought to be substituting for the missing 5,10-

293 methylene-tetrahydromethanopterin reductase $(\mathrm{mer})^{7}$. In contrast, Helarchaeota encode the 
294 canonical archaeal-type mer. To render anaerobic butane oxidation energetically favorable, it

295 must be coupled to the reduction of an electron acceptor such as nitrate, sulfate or iron ${ }^{7,26,27}$. In

296 ANME archaea that lack genes for internal electron acceptors, methane oxidation is enabled

297 through the transfer of electrons to a syntrophic partner organism. In Syntrophoarchaeum,

298 syntrophic butane oxidation is thought to occur through the exchange of electrons via pili and/or

299 cytochromes with sulfate-reducing bacteria ${ }^{7}$. Helarchaeota do not appear to encode any of the

300 systems traditionally associated with syntrophy and no partner was identified in this study. Thus,

301 further research is needed to identify possible bacterial partners.

Furthermore, the hypothesis for Helarchaeota growth through the anaerobic oxidation of

303 short-chain alkanes remains to be confirmed as the genomes of members of this group do not

304 encode canonical routes for electron transfer to a partner bacterium. However, we identified the

305 genetic potential for potential enzymes that may be involved in transfer of electrons. Some

306 methanogenic archaea use formate for syntrophic energy transfer to a syntrophic partner;

307 therefore, the reverse reaction has been speculated to be energetically feasible for methane

308 oxidation ${ }^{27}$. If this is true, the presence of a membrane-bound formate dehydrogenase in the

309 Helarchaeota genomes may support this electron-transferring mechanism, however to our

310 knowledge this has never been shown for an ANME archaea so far. Alternatively, the type 3 NiFe-

311 hydrogenases encoded by Helarchaeota may be involved in transfer of hydrogen to a partner

312 organism. For example, we identified a protein complex distantly related to the $m v h-h d r$ of

313 methanogens for electron transfer (Supplementary material). Mvh-hdr structures have been

314 proposed to be potentially used by non-obligate hydrogenotrophic methanogens for energy

315 transfer, but the directionality of hydrogen exchange could easily be reversed ${ }^{2}$. These 
316 methanogens form syntrophic associations with fermenting, $\mathrm{H}_{2}$-producing bacteria, lack

317 dedicated cytochromes or pili and use the $m v h-h d r$ for electron bifurcation ${ }^{2}$. The detection of a

318 hydrophobic region in the mvh-hdr complex led to the suggestion that this complex could be

319 membrane bound and act as mechanism for electron transfer across the membrane; however, a

320 transmembrane association has never been successfully shown ${ }^{2}$. While the membrane

321 association of the disulfide reductase/FlpD needs to be confirmed, we were able to detect several

322 other transmembrane motifs in the associated proteins that could potentially allow electron

323 transfer in form of hydrogen to an external partner. Thus, while we propose that the most likely

324 explanation for anaerobic short-chain alkane oxidation in Helarchaeota is via a syntrophic

325 interaction with a partner, additional experiments are needed to confirm this working hypothesis.

326 The discovery of alkane-oxidizing pathways and possible syntrophic interactions in a new

327 phylum of Asgard archaea indicates a much wider phylogenetic range for hydrocarbon utilization.

328 Based on their phylogenetic distribution, the Helarchaeota mor operon may have been

329 horizontally transferred from either Bathyarchaeota or Syntrophoarchaeum. However, the

330 preservation of a horizontally transferred pathway indicative of a competitive advantage; it

331 follows that gene transfers among different archaeal phyla reflect alkane oxidation as a desirable

332 metabolic trait. The discovery of the alkyl-CoM reductases and alkane-oxidizing pathways among

333 the Asgard archaea indicates ecological roles for these still cryptic organisms, and opens up a

334 wider perspective on the evolution and expansion of hydrocarbon-oxidizing pathways

335 throughout the archaeal domain. 


\section{Methods}

339 Sample collection and processing. Samples analyzed here are part of a study that aims to characterize

340 the geochemical conditions and microbial community of Guaymas Basin (GB) hydrothermal vent

341 sediments (Gulf of California, Mexico) $)^{31,32}$. The two genomic bins discussed in this paper, Hel_GB_A and

342 Hel_GB_B, were obtained from sediment core samples collected in December 2009 on Alvin dives 4569_2

343 and 4571_4 respectively ${ }^{21}$. Immediately after the dive, freshly recovered sediment cores were separated

344 into shallow $(0-3 \mathrm{~cm})$, intermediate $(12-15 \mathrm{~cm})$ and deep $(21-24 \mathrm{~cm})$ sections for further molecular and

345 geochemical analysis, and frozen at $-80^{\circ} \mathrm{C}$ on the ship until shore-based DNA extraction. Hel_GB_A was

346 recovered from the intermediate sediment $\left(\sim 28^{\circ} \mathrm{C}\right)$ and Hel_GB_B was recovered from shallow sediment

$347\left(\sim 10^{\circ} \mathrm{C}\right)$ from a nearby core (Supplementary Table 1); the sampling context and geochemical gradients of

348 these hydrothermally influenced sediments are published and described in detail ${ }^{21,31}$.

349 DNA was extracted from sediment samples using the MO BIO - PowerMax Soil DNA Isolation kit

350 and sent to the Joint Genome Institute (JGI) for sequencing. A lane of Illumina reads (HiSeq-2500 1TB,

351 read length of $2 \times 151 \mathrm{bp}$ ) was generated for both samples. A total of $226,647,966$ and $241,605,888$ reads

352 were generated for samples from dives for 4569-2 and 4571-4, respectively. Trimmed, screened, paired-

353 end Illumina reads were assembled using the megahit assembler using a range of Kmers (See

354 Supplementary Methods).

356 Genome reconstruction. The contigs from the JGI assembled data were binned using $\mathrm{ESOM}^{33}, \mathrm{MetaBAT}^{34}$

357 and $\mathrm{CONCOCT}^{35}$ and resulting bins were combined using DAS Tool (version 1.0$)^{36}$ (See Supplementary

358 Methods). CheckM lineage_wf (v1.0.5) was run on bins generated from DAS_Tool and 577 bins showed

359 an completeness $>50 \%$ and were characterized further ${ }^{37} .37$ Phylosift $^{38}$ identified marker genes were used

360 for preliminary phylogenetic identification of individual bins (Supplementary Table 6). Thereby, we

361 identified two genomes, belonging to a previously uncharacterized phylum within the Asgard archaea, 
which we named Helarchaeota. To improve the quality of these two Helarchaeota bins (increase the length of the DNA fragments and lower total number), we used Metaspades to reassemble the contigs in each individual bin producing scaffolds. Additionally, we tried to improve the overall assemblies by reassembling the trimmed, screened, paired-end Illumina reads provided by JGI using both IDBA-UD and Metaspades (Supplementary Methods). Binning procedures (using scaffolds longer than $2000 \mathrm{bp}$ ) as previously described in Supplementary Methods for the original bins were repeated with these new assembles. All bins were compared to the original Helarchaeota bins using blastn ${ }^{39}$ for identification. Mmgenome ${ }^{40}$ and CheckM $^{37}$ were used to calculate genome statistics (i.e. contig length, genome size, contamination and completeness). The highest quality Helarchaeota bin from each sample was chosen

371 for further analyses. For the $4572-4$ dataset, the best bin was generated using the Metaspades reassembly

372 on the trimmed data and for the 4569-2 dataset the best bin was recovered using the Metaspades

373 reassembly on the original Hel bin contigs. The final genomes were further cleaned by GC content, paired-

374 end connections, sequence depth and coverage using Mmgenome ${ }^{40}$. CheckM was rerun on cleaned bins

375 to estimate the Hel_GB_A to be $82 \%$ and Hel_GB_B to be $87 \%$ complete and both bins were characterized

376 by a low degree of contamination (between $1.4-2.8 \%$ with no redundancy) (Table 1$)^{37}$. Genome size was

377 estimated to be $4.6 \mathrm{Mbp}$ for Hel_GB_A and 4.1 for Hel_GB_B and was calculated using percent 378 completeness and bin size to extrapolate the likely size of the complete genome. CompareM ${ }^{41}$ was used 379 to analysis differences between Helarchaeota bins and published Asgard bins using the command python comparem aai_wf --tmp_dir tmp/--file_ext fa -c 8 aai_compair_loki aai_compair_loki_output.

16S rRNA gene analysis. Neither bin possessed a $16 \mathrm{~S}$ rRNA gene sequence ${ }^{38}$, and to uncover potentially unbinned 16S rRNA gene sequences from Helarchaeota, all 16S rRNA gene sequences obtained from samples 4569_2 and 4571_4 were identified using JGI-IMG annotations, regardless of whether or not the contig was successfully binned. These $16 \mathrm{~S}$ rRNA gene sequences were compared using blastn ${ }^{39}$ (blastn - 
outfmt 6 -query Hel_possible_16s.fasta -db New_Hel_16s -out Hel_possible_16s_blast.txt -evalue 1E-20)

to newly acquired 16S rRNA gene sequences from MAGs recovered from preliminary data from new GB sites. A 37 Phylosift $^{38}$ marker genes tree was used to assign taxonomy to these MAGs. We were able to identify five MAGs that possessed $16 \mathrm{~s}$ and that formed a monophyletic group with our Hel_GB bins (Supplementary Table 2; Megxx in Figure 2). Of the unbinned 16S rRNA gene sequences one was identified as likely Helarchaeota sequence. The contig was retrieved from the 4572 _4 assembly (designated Ga0180301_10078946) and was 2090 bp long and encoded for an 16S rRNA gene sequence that was 1058 bp long. Given the small size of this contig relative to the length of the 16S rRNA gene none of the other 394 genes on the contig could be annotated. Blastn ${ }^{39}$ comparison to published Asgard $16 \mathrm{~S}$ rRNA gene sequences was performed using the following command: blastn -outfmt 6 -query Hel_possible_16s.fasta -db Asgrad_16s -out Hel_possible_16s_blast.txt -evalue 1E-20 (Supplementary Table 2). The GC content of each 16S rRNA gene sequence was calculated using the Geo-omics script length+GC.pl (https://github.com/Geo-omics/scripts/blob/master/AssemblyTools/length\%2BGC.pl). For a further phylogenetic placement, the 16S rRNA gene sequences were aligned to the SILVA database (SINA v1.2.11) using the SILVA online server ${ }^{42}$ and Geneious $(\mathrm{v} 10.1 .3)^{43}$ was used to manually trim sequences. The alignment also contained 16S rRNA gene sequences from the new, preliminary Helarchaeota bins. The cleaned alignment was used to generated a maximum-likelihood tree with RAxML as follows: "/raxmIHPCPTHREADS-AVX -T 20 -f a -m GTRGAMMA -N autoMRE -p 12345 -x 12345 -s Nucleotide_alignment.phy -n output" (Figure 1b).

Phylogenetic analysis of ribosomal proteins. For a more detailed phylogenetic placement, we used

407 BLASTp ${ }^{44}$ to identify orthologs of 56 ribosomal proteins in the two Helarchaeota bins, as well as from a 408 selection of 130 representative taxa of archaeal diversity and 14 eukaryotes. The full list of marker genes 
410 aligned using mafft-linsi ${ }^{45}$ and ambiguously aligned positions were trimmed using BMGE (-m BLOSUM30) ${ }^{46}$.

411 Maximum likelihood (ML) individual phylogenies were reconstructed using IQtree v. $1.5 .5^{47}$ under the

412 LG+C20+G substitution model with 1000 ultrafast bootstraps that were manually inspected. Trimmed

413 alignments were concatenated into a supermatrix, and two additional datasets were generated by

414 removing eukaryotic and/or DPANN homologues to test the impact of taxon sampling on phylogenetic

415 reconstruction. For each of these concatenated datasets, phylogenies were inferred using $\mathrm{ML}$ and

416 Bayesian approaches. $\mathrm{ML}$ phylogenies were reconstructed using IQtree under the $\mathrm{LG}+\mathrm{C} 60+\mathrm{F}+\mathrm{G}+\mathrm{PMSF}$

417 model $^{48}$. Statistical support for branches was calculated using 100 bootstraps replicated under the same

418 model. To test robustness of the phylogenies, the dataset was subjected to several treatments. For the

419 'full dataset' (i.e., with all 146 taxa), we tested the impact of removing the $25 \%$ fastest-evolving sites, as

420 within a deep phylogenetic analysis, these sites are often saturated with multiple substitutions and, as a

421 result of model-misspecification can manifest in an artifactual signal ${ }^{50-52}$. The corresponding ML tree was

422 inferred as described above. Bayesian phylogenies were reconstructed with Phylobayes for the dataset

423 "without DPANN" under the LG+GTR model. Four independent Markov chain Monte Carlo chains were

424 run for $\sim 38,000$ generations. After a burn-in of $20 \%$, convergence was achieved for three of the chains

425 (maxdiff $<0.29$ ). The initial supermatrix was also recoded into 4 categories, in order to ameliorate effects

426 of model misspecification and saturation ${ }^{52}$ and the corresponding phylogeny was reconstructed with

427 Phylobayes, under the CAT+GTR model. Four independent Markov chain Monte Carlo chains were run

428 for $\sim 49,000$ generations. After a burn-in of 20 convergence was achieved for all four the chains (maxdiff

$429<0.19)$. All phylogenetic analyses performed are summarized in Supplementary Table 8, including maxdiff

430 values and statistical support for the placement of Helarchaeota, and of eukaryotes.

431

432 Phylogenetic analysis of McrA and concatenated McrA and McrB proteins. McrA homologs were aligned

433 using mafft-linsi ${ }^{45}$, trimmed with trim $\mathrm{AL}^{53}$ and the final alignment consisting of 528 sites was subjected to 
434 phylogenetic analyses using v. $1.5 .5^{47}$ with the $L G+C 60+R+F$ model. Support values were estimated using

4351000 ultrafast boostraps ${ }^{54}$ and $\mathrm{SH}$-like approximate likelihood ratio test ${ }^{55}$, respectively. Sequences for

436 McrA and B were aligned separately with mafft-linsi ${ }^{45}$ and trimmed using trimAL Subsequently, McrA and

437 McrB encoded in the same gene cluster, were concatenated yielding a total alignment of 972 sites.

438 Bayesian and Maximum likelihood phylogenies were inferred using IQtree v. $1.5 .5^{47}$ with the mixture

439 model LG+C60+R+F and PhyloBayes v. $3.2^{56}$ using the CAT-GTR model. For Maximum likelihood inference,

440 support values were estimated using 1000 ultrafast boostraps ${ }^{54}$ and $\mathrm{SH}$-like approximate likelihood ratio

441 test ${ }^{55}$, respectively. For Bayesian analyses, four chains were run in parallel, sampling every 50 points until

442 convergence was reached (maximum difference $<0.07$; mean difference $<0.002$ ). The first $25 \%$ or the

443 respective generations were selected as burn-in. Phylobayes posterior predictive values were mapped

444 onto the IQtree using sumlabels from the DendroPy package ${ }^{57}$. The final trees were rooted artificially

445 between the canonical Mcr and divergent Mcr-like proteins, respectively.

447 Metabolic Analyses. Gene prediction for the two Helarchaeota bins was performed using prodigal ${ }^{58}$ 448 (V2.6.2) with default settings and Prokka ${ }^{59}$ (v1.12) with the extension '-kingdom archaea'. Results for both 449 methods were comparable and yielded a total of 3,574-3,769 and 3,164-3,287 genes for Hel_GB_A and 450 Hel_GB_B, respectively, with Prokka consistently identifying fewer genes. Genes were annotated by 451 uploading the protein fasta files from both methods to KAAS (KEGG Automatic Annotation Server) for 452 complete or draft genomes to assign orthologs $s^{60}$. Files were run using the following settings: prokaryotic 453 option, GhostX and bi-directional best hit $(\mathrm{BBH})^{60}$. Additionally, genes were annotated by JGI-IMG ${ }^{61}$ to 454 confirm hits using two independent databases. Hits of interest were confirmed using blastp on the NCBI 455 webserver ${ }^{44}$. The $d b C A N^{62}$ and MEROPS ${ }^{63}$ webserver were run using default conditions for identification 456 of carbohydrate degrading enzymes and peptidases respectively. Hits with e-values lower than $\mathrm{e}^{\wedge}-20$ were 
discarded. In addition to these methods an extended search was used to categorize genes involved in

458 butane metabolism, syntrophy and energy transfer.

Identified genes predicted to code for putative alkane oxidation proteins were similar to those described from Candidatus Syntrophoarchaeum spp.. Therefore, a blastp ${ }^{44}$ database consisting of proteins

461 predicted to be involved in the alkane oxidation pathway of $\mathrm{Ca}$. Syntrophoarchaeum was created in order

462 to identify additional proteins in Helarchaeota, which may function in alkane oxidation. Positive hits were

463 confirmed with blastp ${ }^{44}$ on the NCBI webserver and compared to the annotations from JGI-IMG ${ }^{61}$, 464 Interpro ${ }^{64}$, PROKKA $^{59}$ and KAAS $^{60}$ annotation. Genes for mcrABG were further confirmed by a HMMER ${ }^{65}$ 465 search to a published database using the designated threshold values ${ }^{66}$ and multiple MCR trees (see 466 Methods). To confirm that the contigs with the mcrA gene cluster were not missbined, all other genes on 467 these contigs were analyzed for their phylogenetic placement and gene content. The prodigal protein 468 predictions for genes on the contigs with mcrA operons were used to determine directionality and length 469 of the potential operon.

To identify genes that are involved in electron and hydrogen transfer across the membrane, a

471 database was created of known genes relevant in syntrophy that were download from NCBI. The protein 472 sequences of the two Helarchaeota genomes were blasted against the database to detect relevant hits

473 (E-value $\geq \mathrm{e}^{\wedge}-10$ ). All hits were confirmed using the NCBI webserver, Interpro, JGI-IMG and KEGG.

474 Hydrogenases were identified by a HMMER search to published database using the designated threshold 475 values $^{67}$. Hits were confirmed with comparisons against JGI annotations and NCBI blasts, the HydDB 476 database ${ }^{68}$ and a manual database made from published sequences ${ }^{69,70}$. All detected hydrogenases were 477 used to generate two phylogenetic trees, one for proteins identified as small subunits and one for large 478 subunits in order to properly identify the different hydrogenase subgroups. Hydrogenases that are part 479 of the proposed complex were then further analyzed to evaluate if this was a possible operon by looking 480 for possible transcription factors and binding motifs (Supplementary Methods). 
482 ESP Identification. Gene prediction for the two Helarchaeota bins was performed using prodigal ${ }^{58}(\mathrm{~V} 2.6 .2)$

483 with default settings. All the hypothetical proteins inferred in both Helarchaeaota were used as seeds

484 against InterPro ${ }^{64}$, arCOG ${ }^{71}$ and $\mathrm{nr}$ using BLAST ${ }^{44}$. The annotation table from Zaremba-Niedzwiedzka, et al.

485 2017. was used as a basis for the comparison ${ }^{12}$. The IPRs (or in some cases, the arCOGs) listed in the

486 Zaremba-Niedzwiedzka, et al. 2017 were searched for in the Helarchaeota genomes ${ }^{12}$ and the resulting

487 information was used to complete the presence/absence table. When something that had previously been

488 detected in an Asgard bin was not found in a Helarchaeota bin using the InterPro/arCOG annotations,

489 BLASTs were carried out using the closest Asgard seeds to verify the absence. In some cases, specific

490 analyses were used to verify the homology or relevance of particular sequences. The details for each

491 individual ESP are depicted in supplementary materials.

492

493 Data Availability. The raw reads from the metagenomes described in this study are available at JGI under

494 the IMG genome ID 3300014911 and 3300013103 for samples 4569-2 and 4571-4, respectively. Genome

495 sequences are available at NCBI under the accession numbers SAMN09406154 and SAMN09406174 for

496 Hel_GB_A and Hel_GB_B respectively. Both are associated with BioProject PRJNA362212. 


\section{Tables}

506

507

508

509

510

511

512

513

514

515

516

517

518

519

520

Table 1. Bin statistics for Helarchaeota Bins. Degree of completeness, contamination and heterogeneity was determined using CheckM ${ }^{37}$.

\begin{tabular}{|l|c|c|}
\hline SeqID & Hel_GB_A & Hel_GB_B \\
\hline Completeness (\%) & 82.4 & 86.92 \\
\hline Contamination (\%) & 2.8 & 1.40 \\
\hline Strain heterogeneity (\%) & 0 & 0 \\
\hline Scaffold number & 333 & 182 \\
\hline GC content (\%) & 35.40 & 28.00 \\
\hline N50 (bp) & 15,161 & 28,908 \\
\hline Length total (Mbp) & 3.84 & 3.54 \\
\hline Estimated Genome size (Mbp) & 4.6 & 4.1 \\
\hline Longest contig (bp) & 52,512 & 72,379 \\
\hline Mean contig (bp) & 11,531 & 19,467 \\
\hline
\end{tabular}

1. G E Claypool \& Kvenvolden, and K. A. Methane and other Hydrocarbon Gases in Marine Sediment. Annu. Rev. Earth Planet. Sci. 11, 299-327 (1983).

2. Thauer, R. K., Kaster, A.-K., Seedorf, H., Buckel, W. \& Hedderich, R. Methanogenic archaea: ecologically relevant differences in energy conservation. Nat. Rev. Microbiol. 6, 579-591 (2008).

3. Reeburgh, W. S. Oceanic Methane Biogeochemistry. Chem. Rev. 107, 486-513 (2007).

4. Spang, A., Caceres, E. F. \& Ettema, T. J. G. Genomic exploration of the diversity, ecology, and evolution of the archaeal domain of life. Science 357, eaaf3883 (2017).

5. Evans, P. N. et al. Methane metabolism in the archaeal phylum Bathyarchaeota revealed by genome-centric metagenomics. Science 350, 434-438 (2015). 
521 6. Vanwonterghem, I. et al. Methylotrophic methanogenesis discovered in the archaeal phylum

522 Verstraetearchaeota. Nat. Microbiol. 1, 16170 (2016).

523 7. Laso-Pérez, R. et al. Thermophilic archaea activate butane via alkyl-coenzyme M formation.

$524 \quad$ Nature 539, 396-401 (2016).

525 8. Dombrowski, N., Seitz, K. W., Teske, A. P. \& Baker, B. J. Genomic insights into potential

526 interdependencies in microbial hydrocarbon and nutrient cycling in hydrothermal sediments.

$527 \quad$ Microbiome 5, 106 (2017).

528 9. Bazylinski, D. A., Farrington, J. W. \& Jannasch, H. W. Hydrocarbons in surface sediments from 529 a Guaymas Basin hydrothermal vent site. Org. Geochem. 12, 547-558 (1988).

530 10. Teske, A., Callaghan, A. V. \& LaRowe, D. E. Biosphere frontiers of subsurface life in the 531 sedimented hydrothermal system of Guaymas Basin. Front. Microbiol. 5, (2014).

532 11. Von Damm, K. L., Edmond, J. M., Measures, C. I. \& Grant, B. Chemistry of submarine 533 hydrothermal solutions at Guaymas Basin, Gulf of California. Geochim. Cosmochim. Acta 49, $534 \quad 2221-2237(1985)$.

535 12. Zaremba-Niedzwiedzka, K. et al. Asgard archaea illuminate the origin of eukaryotic 536 cellular complexity. Nature 541, 353-358 (2017).

537 13. Spang, A. et al. Complex archaea that bridge the gap between prokaryotes and 538 eukaryotes. Nature 521, 173-179 (2015).

539 14. Seitz, K. W., Lazar, C. S., Hinrichs, K.-U., Teske, A. P. \& Baker, B. J. Genomic

540 reconstruction of a novel, deeply branched sediment archaeal phylum with pathways for 541 acetogenesis and sulfur reduction. ISME J 10, 1696-1705 (2016). 
$54215 . \quad J \emptyset r g e n s e n$, S. L., Thorseth, I. H., Pedersen, R. B., Baumberger, T. \& Schleper, C.

543 Quantitative and phylogenetic study of the Deep Sea Archaeal Group in sediments of the

$544 \quad$ Arctic mid-ocean spreading ridge. Front. Microbiol. 4, (2013).

545 16. Jorgensen, S. L. et al. Correlating microbial community profiles with geochemical data in 546 highly stratified sediments from the Arctic Mid-Ocean Ridge. Proc. Natl. Acad. Sci. U. S. A. 547 109, E2846-2855 (2012).

548 17. Hartman, H. \& Fedorov, A. The origin of the eukaryotic cell: a genomic investigation. 549 Proc. Natl. Acad. Sci. 99, 1420-1425 (2002).

550 18. Eme, L., Spang, A., Lombard, J., Stairs, C. \& J. G. Ettema, T. Archaea and the origin of $551 \quad$ eukaryotes. 15, (2017).

552 19. Spang, A. et al. A renewed syntrophy hypothesis for the origin of the eukaryotic cell 553 based on comparative analysis of Asgard archaeal metabolism. Nat. Microbiol. Submitted,

554 20. Dombrowski, N., Teske, A. P. \& Baker, B. J. Extensive metabolic versatility and 555 redundancy in microbially diverse, dynamic Guaymas Basin hydrothermal sediments. Nat.

556 Commun. 9:4999, (2018).

557 21. McKay, L. et al. Thermal and geochemical influences on microbial biogeography in the 558 hydrothermal sediments of Guaymas Basin, Gulf of California. Environ. Microbiol. Rep. 8, $559 \quad$ 150-161 (2016).

560 22. Yarza, P. et al. Uniting the classification of cultured and uncultured bacteria and archaea 561 using 16S rRNA gene sequences. Nat. Rev. Microbiol. 12, 635-645 (2014).

562 23. Lazar, C. S. et al. Environmental controls on intragroup diversity of the uncultured 563 benthic archaea of the miscellaneous Crenarchaeotal group lineage naturally enriched in 

anoxic sediments of the White Oak River estuary (North Carolina, USA). Environ. Microbiol. $17,2228-2238(2015)$.

24. Tabita, F. R., Satagopan, S., Hanson, T. E., Kreel, N. E. \& Scott, S. S. Distinct form I, II, III,

567 and IV Rubisco proteins from the three kingdoms of life provide clues about Rubisco

568 evolution and structure/function relationships. J. Exp. Bot. 59, 1515-1524 (2007).

569 25. Dowell, F. et al. Microbial Communities in Methane- and Short Chain Alkane-Rich

$570 \quad$ Hydrothermal Sediments of Guaymas Basin. Front. Microbiol. 7, (2016).

$57126 . \quad$ Krukenberg, V. et al. Candidatus Desulfofervidus auxilii, a hydrogenotrophic sulfate-

572 reducing bacterium involved in the thermophilic anaerobic oxidation of methane. Environ.

$573 \quad$ Microbiol. 18, 3073-3091 (2016).

574 27. Stams, A. J. M. \& Plugge, C. M. Electron transfer in syntrophic communities of anaerobic 575 bacteria and archaea. Nat. Rev. Microbiol. 7, 568-577 (2009).

576 28. Meuer, J., Kuettner, H. C., Zhang, J. K., Hedderich, R. \& Metcalf, W. W. Genetic analysis 577 of the archaeon Methanosarcina barkeri Fusaro reveals a central role for Ech hydrogenase 578 and ferredoxin in methanogenesis and carbon fixation. Proc. Natl. Acad. Sci. 99, 5632-5637 579 (2002).

580 29. Kunow, J., Linder, D., Stetter, K. O. \& Thauer, R. K. F420H2: quinone oxidoreductase 581 from Archaeoglobus fulgidus. Eur. J. Biochem. 223, 503-511 (1994).

582 30. Wegener, G., Krukenberg, V., Riedel, D., Tegetmeyer, H. E. \& Boetius, A. Intercellular 583 wiring enables electron transfer between methanotrophic archaea and bacteria. Nature 526, $584 \quad 587-590$ (2015). 
585

586

587

588

589

590

591

592

593

594

595

596

597

598

599

600

601

602

603

604

605

606

31. McKay, L. J. et al. Spatial heterogeneity and underlying geochemistry of phylogenetically diverse orange and white Beggiatoa mats in Guaymas Basin hydrothermal sediments. Deep Sea Res. Part Oceanogr. Res. Pap. 67, 21-31 (2012).

32. Meyer, S. et al. Microbial habitat connectivity across spatial scales and hydrothermal temperature gradients at Guaymas Basin. Front. Microbiol. 4, (2013).

33. Dick, G. J. et al. Community-wide analysis of microbial genome sequence signatures. Genome Biol. 10, R85 (2009).

34. Kang, D. D., Froula, J., Egan, R. \& Wang, Z. MetaBAT, an efficient tool for accurately reconstructing single genomes from complex microbial communities. PeerJ 3, e1165 (2015).

35. Alneberg, J. et al. Binning metagenomic contigs by coverage and composition. Nat. Methods 11, nmeth.3103 (2014).

36. Sieber, C. M. K. et al. Recovery of genomes from metagenomes via a dereplication, aggregation, and scoring strategy. bioRxiv 107789 (2017). doi:10.1101/107789

37. Parks, D. H., Imelfort, M., Skennerton, C. T., Hugenholtz, P. \& Tyson, G. W. CheckM: assessing the quality of microbial genomes recovered from isolates, single cells, and metagenomes. Genome Res. gr.186072.114 (2015). doi:10.1101/gr.186072.114

38. Darling, A. E. et al. PhyloSift: phylogenetic analysis of genomes and metagenomes. PeerJ 2, e243 (2014).

39. Altschul, S. F., Gish, W., Miller, W., Myers, E. W. \& Lipman, D. J. Basic local alignment search tool. J. Mol. Biol. 215, 403-410 (1990).

40. Karst, S. M., Kirkegaard, R. H. \& Albertsen, M. mmgenome: a toolbox for reproducible genome extraction from metagenomes. bioRxiv 059121 (2016). doi:10.1101/059121 
607 41. https://github.com/dparks1134/CompareM.

608 42. Pruesse, E., Peplies, J. \& Glöckner, F. O. SINA: Accurate high-throughput multiple

609 sequence alignment of ribosomal RNA genes. Bioinformatics 28, 1823-1829 (2012).

610 43. Kearse, M. et al. Geneious Basic: an integrated and extendable desktop software

611 platform for the organization and analysis of sequence data. Bioinforma. Oxf. Engl. 28, 16476121649 (2012).

613 44. Altschul, S. F. et al. Gapped BLAST and PSI-BLAST: a new generation of protein database 614 search programs. Nucleic Acids Res. 25, 3389-3402 (1997).

615 45. Katoh, K. \& Standley, D. M. MAFFT multiple sequence alignment software version 7:

616 Improvements in performance and usability. Mol. Biol. Evol. 30, 772-780 (2013).

617 46. Criscuolo, A. \& Gribaldo, S. BMGE (Block Mapping and Gathering with Entropy): a new 618 software for selection of phylogenetic informative regions from multiple sequence 619 alignments. BMC Evol. Biol. 10, 210 (2010).

620 47. Nguyen, L.-T., Schmidt, H. A., von Haeseler, A. \& Minh, B. Q. Iq-tree: A fast and effective 621 stochastic algorithm for estimating maximum-likelihood phylogenies. Mol. Biol. Evol. 32, $622 \quad 268-274(2015)$.

623 48. Wang, H.-C., Minh, B. Q., Susko, E. \& Roger, A. J. Modeling Site Heterogeneity with 624 Posterior Mean Site Frequency Profiles Accelerates Accurate Phylogenomic Estimation. Syst. 625 Biol. syx068 (2017).

626 49. Jeffroy, O., Brinkmann, H., Delsuc, F. \& Philippe, H. Phylogenomics: the beginning of 627 incongruence? Trends Genet. 22, 225-231 (2006). 
628 50. Lartillot, N. \& Philippe, H. Improvement of molecular phylogenetic inference and the 629 phylogeny of Bilateria. Philos. Trans. R. Soc. Lond. B. Biol. Sci. 363, 1463-72 (2008).

630 51. Brown, M. W. M. et al. Phylogenomics demonstrates that breviate flagellates are related 631 to opisthokonts and apusomonads. Proc. R. Soc. B Biol. Sci. 280, 20131755 (2013).

632 52. Susko, E. \& Roger, A. J. On reduced amino acid alphabets for phylogenetic inference. 633 Mol. Biol. Evol. 24, 2139-2150 (2007).

634 53. Capella-Gutiérrez, S., Silla-Martínez, J. M. \& Gabaldón, T. trimAl: a tool for automated 635 alignment trimming in large-scale phylogenetic analyses. Bioinforma. Oxf. Engl. 25, 1972$6361973(2009)$.

637 54. Minh, B. Q., Nguyen, M. A. T. \& von Haeseler, A. Ultrafast approximation for 638 phylogenetic bootstrap. Mol. Biol. Evol. 30, 1188-1195 (2013).

639 55. Guindon, S. et al. New algorithms and methods to estimate maximum-likelihood 640 phylogenies: assessing the performance of PhyML 3.0. Syst. Biol. 59, 307-321 (2010).

641 56. Lartillot, N. \& Philippe, H. A Bayesian mixture model for across-site heterogeneities in 642 the amino-acid replacement process. Mol. Biol. Evol. 21, 1095-1109 (2004).

643 57. Sukumaran, J. \& Holder, M. T. DendroPy: a Python library for phylogenetic computing. 644 Bioinforma. Oxf. Engl. 26, 1569-1571 (2010).

645 58. Hyatt, D. et al. Prodigal: prokaryotic gene recognition and translation initiation site 646 identification. BMC Bioinformatics 11, 119 (2010).

647 59. Seemann, T. Prokka: rapid prokaryotic genome annotation. Bioinforma. Oxf. Engl. 30, $648 \quad$ 2068-2069 (2014). 
649 60. Moriya, Y., Itoh, M., Okuda, S., Yoshizawa, A. C. \& Kanehisa, M. KAAS: an automatic 650 genome annotation and pathway reconstruction server. Nucleic Acids Res. 35, W182-W185 651 (2007).

652 61. Markowitz, V. M. et al. IMG: the integrated microbial genomes database and 653 comparative analysis system. Nucleic Acids Res. 40, D115-D122 (2012).

654 62. Yin, Y. et al. dbCAN: a web resource for automated carbohydrate-active enzyme 655 annotation. Nucleic Acids Res. 40, W445-W451 (2012).

656 63. Rawlings, N. D., Barrett, A. J. \& Bateman, A. MEROPS: the peptidase database. Nucleic 657 Acids Res. 38, D227-D233 (2010).

658 64. Jones, P. et al. InterProScan 5: genome-scale protein function classification. Bioinforma. Oxf. Engl. 30, 1236-1240 (2014).

65. Johnson, L. S., Eddy, S. R. \& Portugaly, E. Hidden Markov model speed heuristic and iterative HMM search procedure. BMC Bioinformatics 11, 431 (2010).

66. Anantharaman, K. et al. Thousands of microbial genomes shed light on interconnected biogeochemical processes in an aquifer system. Nat. Commun. 7, 13219 (2016).

67. Anantharaman, K. et al. Thousands of microbial genomes shed light on interconnected biogeochemical processes in an aquifer system. 7, (2016).

68. Søndergaard, D., Pedersen, C. N. S. \& Greening, C. HydDB: A web tool for hydrogenase classification and analysis. Sci. Rep. 6, 34212 (2016).

668 69. Vignais, P. M. \& Billoud, B. Occurrence, classification, and biological function of 669 hydrogenases: an overview. Chem. Rev. 107, 4206-4272 (2007). 
$67070 . \quad$ Vignais, P. M., Billoud, B. \& Meyer, J. Classification and phylogeny of hydrogenases1.

671 FEMS Microbiol. Rev. 25, 455-501

672 71. Makarova, K. S., Wolf, Y. I. \& Koonin, E. V. Archaeal Clusters of Orthologous Genes

673 (arCOGs): An Update and Application for Analysis of Shared Features between

674 Thermococcales, Methanococcales, and Methanobacteriales. Life 5, 818-840 (2015).

675

676

677 Acknowledgements

678 This study was supported in part by an Alfred P. Sloan Foundation fellowship (FG-2016-6301) and

679 National Science Foundation Directorate of Biological Sciences (award 1737298) to BJB. Sampling 680 in Guaymas Basin and post-cruise work was supported by NSF Awards OCE-0647633 and OCE6811357238 to APT, respectively. The work conducted by the U.S. Department of Energy Joint 682 Genome Institute, a DOE Office of Science User Facility, is supported by the Office of Science of 683 the U.S. Department of Energy under Contract No. DE-AC02-05CH11231 provided to ND.

\section{Author contributions}

686 KWS, TJGE, ND and BJB conceived the study. KWS, ND, and BJB analyzed the genomic data. APT 687 collected and processed samples. KWS, AS, and LE performed phylogenetic analyses. JL analyzed 688 ESPS. KWS, AS, JRS, APT, BJB handled the metabolic inferences. BJB and KWS wrote the 689 manuscript with inputs from all authors. 